\title{
Dark Matter Spikes and Indirect Detection
}

\author{
David Merritt \\ Rutgers University, New Brunswick, NJ 08903, USA \\ E-mail: merritt@physics.rutgers.edu
}

\begin{abstract}
Annihilation radiation from supersymmetric particles at the Galactic center could be greatly enhanced if the dark matter density is peaked around the supermassive black hole. Arguments for and against the existence of density spikes are reviewed. Spikes are destroyed during mergers, and there is strong evidence for this effect in stellar density profiles. The dark matter spike at the Galactic center probably suffered this fate.
\end{abstract}

Indirect detection schemes are based on searches for gamma rays, neutrinos or other annihilation by-products from supersymmetric particles in the dark matter halo of the Milky Way [1]. The flux depends on the squared density of particles integrated along the line of sight and the signal is greatly enhanced in directions where the dark matter is clumped. This includes the center of the Milky Way where the density in a smooth halo would be maximum [2, 3]. The signal from the Galactic center is further enhanced if there is a dark matter "spike" associated with the central supermassive black hole (SBH) 4. Growth of a SBH causes the orbits of nearby dark matter particles to shrink and their density to rise; for a wide range of initial conditions, the resulting profile is a steep power law, $\rho \sim r^{-2}$, implying a formally divergent annihilation flux from near the $\mathrm{SBH}[4]$.

This article reviews the arguments for and against the existence of dark matter density spikes. A useful guide to the distribution of dark matter at the very centers of galaxies is the stellar distribution; in the absence of gaseous dissipation, stars act like a nearly collisionless fluid and should react to the presence of a SBH in the same way as the dark matter. Steep spikes in the stellar luminosity profile are only seen in galaxies which show evidence of dissipative formation. Other galaxies exhibit shallow inner profiles, and this is consistent with a model in which binary SBHs injected energy into the stellar fluid during the mergers that formed the galaxies. Mergers almost certainly occurred during the formation of SBHs and their host bulges, implying destruction of the dark matter spikes.

\section{The Adiabatic Growth Model}

If a black hole grows at the center of a collisionless fluid, the density around it also grows as the black hole's gravity causes the surrounding orbits to shrink. The result is 

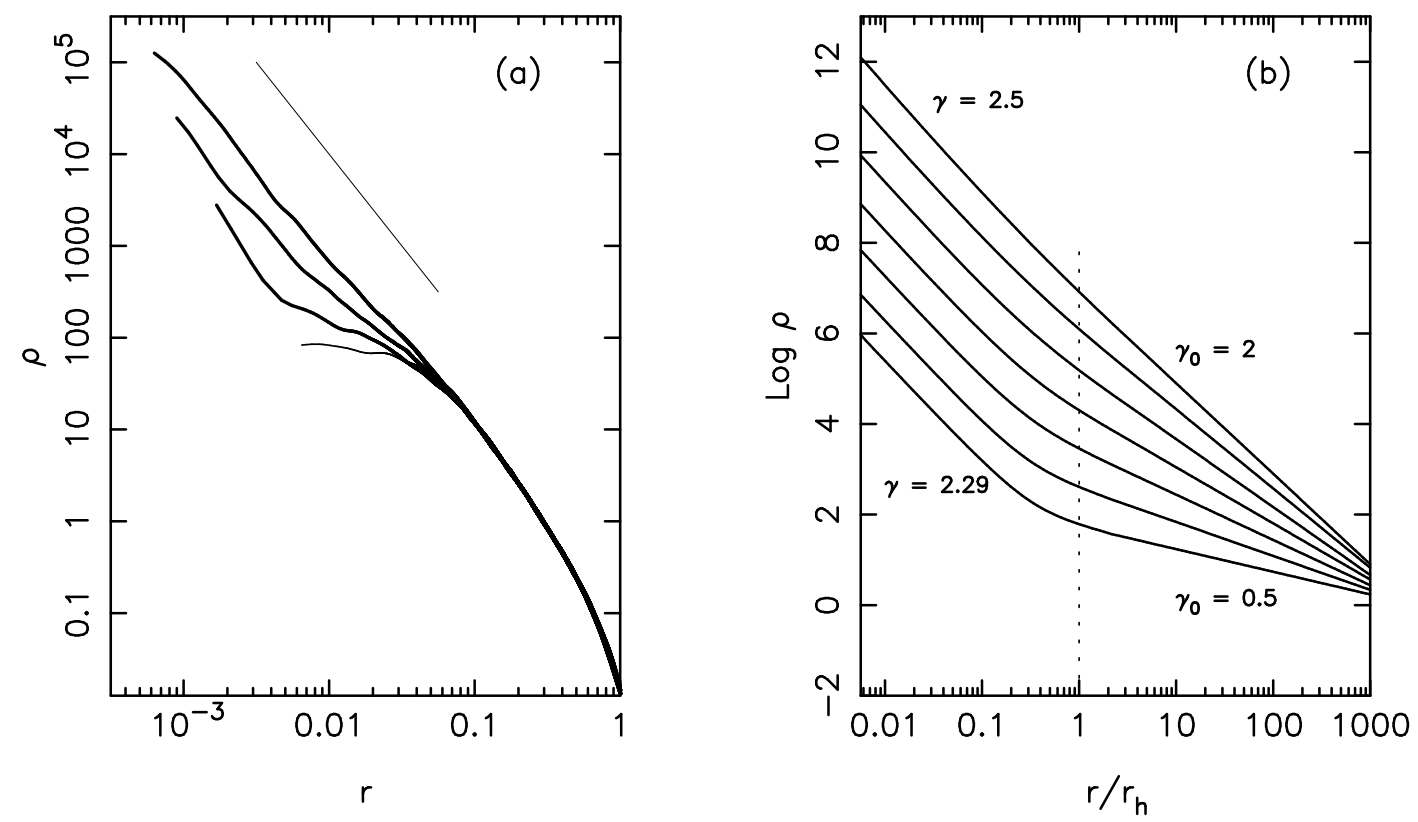

Figure 1. Spike formation by adiabatic growth of a black hole. (a) Thin curve is the initial model, formed by collapse in an $N$-body simulation. Heavy curves are density profiles after growth of a central "black hole" containing $0.3 \%, 1 \%$ and $3 \%$ of the total mass. The reference line has a logarithmic slope of -2 . (From Ref. 5.) (b) Initial models were spherical isotropic halos with power-law density profiles, $\rho \propto r^{-\gamma_{0}} ; \gamma_{0}$ increases upwards in steps of 0.25 . The radial scale is normalized to $r_{h}$ in the initial halo. The slope of the final profile at $r \lesssim r_{h}$ is almost independent of the initial slope.

simplest to compute under the "adiabatic" approximation in which the growth time is long compared with orbital periods; this is reasonable in the case of SBHs at galactic centers, whose growth time is thought to be $\sim 10^{8} \mathrm{yr}$ compared with orbital periods of $\sim 10^{6} \mathrm{yr}$. The resulting density profile depends somewhat on the initial state. One possibility is a constant-density, isothermal core; the resulting spike is a power law, $\rho \propto r^{-1.5}, r \lesssim r_{h} \equiv G M_{\bullet} / \sigma^{2}$, with $M_{\bullet}$ the final black hole mass and $\sigma$ the 1D particle velocity dispersion before the appearance of the black hole [5, 6].

A more reasonable guess for the initial profile is a power law, $\rho \propto r^{-\gamma_{0}}$; power-law central profiles are generic outcomes of hierarchical structure formation simulations [8, 9], although the index of the power law is debated. Adiabatic growth in a power law gives a final profile that is also a power law near the black hole but with steeper index[4]:

$$
\rho_{f}(r) \propto r^{-\gamma}, \quad \gamma=2+\frac{1}{4-\gamma_{0}} \quad\left(0<\gamma_{0}<2\right) .
$$

The final index is almost independent of the initial index: $2.25<\gamma<2.5$ (Figure 1). Other reasonable initial profiles can be found[10] that generate final profiles which fill the gap between the power laws and the isothermal sphere. Hence in the absence of detailed knowledge about the initial state, the adiabatic growth model predicts density profiles such that

$$
\rho_{f}(r) \propto r^{-\gamma}, \quad 1.5 \lesssim \gamma \lesssim 2.5 .
$$


Even slopes at the low end of this range should not be ruled out since the latest simulations [11] suggest that $\gamma_{0}$ may decline monotonically toward the center. Essentially nothing is known about the structure of CDM halos on the very small $(\lesssim 100 \mathrm{pc})$ scales relevant to the formation of a spike 12 .

\section{Stellar Density Profiles}

The predictions of the adiabatic growth model can be tested against stellar luminosity profiles in galactic nuclei. The time scales for both physical collisions and gravitational near-encounters exceed a Hubble time for the stars in most nuclei[13; in the absence of ongoing star formation, nuclei should have retained whatever density profiles were set up when the SBH gained its current size. Observed profiles are indeed well described as power laws within the SBH's sphere of influence, but with slopes that vary systematically as a function of galaxy luminosity [14, 15]. Faint ellipticals and bulges, $M_{V} \gtrsim-20$, exhibit roughly the range of slopes predicted by the adiabatic growth model, $1.5 \lesssim \gamma \lesssim 2.5$. However bright galaxies have shallower inner profiles, $0 \lesssim \gamma \lesssim 1.5$.

There are problems with identifying even the steep profiles in faint galaxies with the adiabatic growth picture[16. Spikes produced by adiabatic growth almost always exhibit an inflection at $r \sim r_{h}$ (Figure 1); the logarithmic slope increases inwards. In real galaxies, the slope decreases inwards; inflections are virtually never seen, except at much smaller radii where they are associated with point-like or non-thermal nuclei. Avoiding an inflection requires fine-tuning of the initial conditions: either the initial profile has to be steep, $\gamma_{0} \approx 2$, which obviates the need for adiabatic growth; or the $\mathrm{SBH}$ has to be just the right size that the pre-existing slope at $r \sim r_{h}$ matches the final slope $\gamma$.

The rapid rotation, high densities and disky isophotes of low-luminosity ellipticals and bulges imply that gas dynamics played a dominant role in their formation [17. The steep stellar density spike at the center of the Milky Way, $\rho_{*} \sim r^{-2}$, may be the result of sustained star formation from a reservoir of dense molecular gas [18]. Semi-analytic models of galaxy formation [19] suggest that galaxies fainter than $M_{V} \sim-20$ were formed from gas-rich progenitors. These arguments suggest that the structure of nuclei in faint elliptical galaxies and bulges is a combined result of SBH growth and gas dynamics. For this reason, faint galaxies are not ideal testing grounds for the adiabatic growth model.

A cleaner test of the model comes from bright elliptical galaxies, and here the model clearly fails: nuclear profiles in bright galaxies are almost always flatter than the minimum slope allowed by adiabatic growth, $\gamma \sim 1.5$. Profiles are sometimes so flat that they are best described as "cores," regions of nearly-constant density; indeed central minima have been claimed in a handful of galaxies [20]. Core radii can be $\sim 10^{2}$ pc or more. Something other than adiabatic SBH growth is required to explain this structure. 


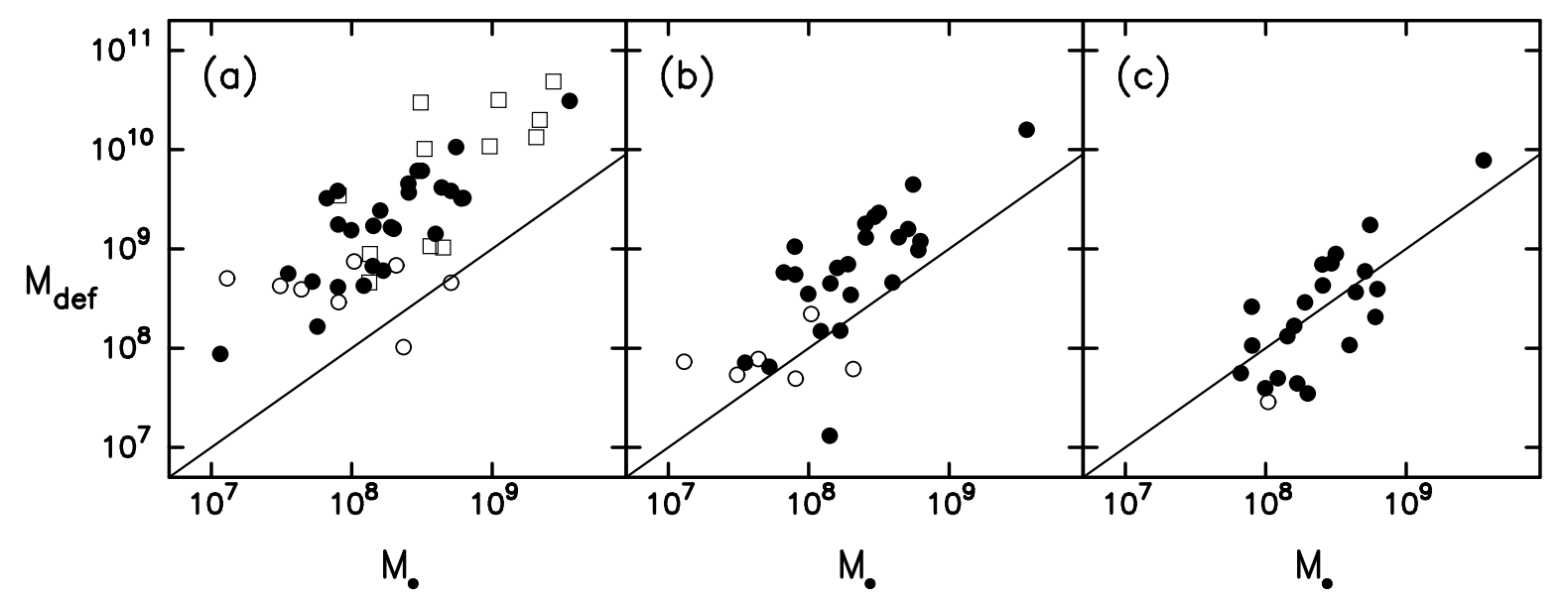

Figure 2. Mass deficit vs SBH mass for three different assumed values of $\gamma_{0}$, the assumed logarithmic slope of the density cusp before changes due to ejection of stars by a binary SBH. (a) $\gamma_{0}=2$; (b) $\gamma_{0}=1.75$; (c) $\gamma_{0}=1.5$. Solid lines are $M_{\text {def }}=M_{\bullet}$; units are solar masses. (From Ref. 26.)

\section{The Binary Black Hole Model}

A natural way to explain the low central densities of bright galaxies is mergers. A SBH is effective at tidally disrupting the density cusp of an infalling galaxy [21, 22]. If the latter also contains a SBH, a binary SBH will form, which ejects passing stars or dark matter particles via the "gravitational slingshot." 23, 24] A binary SBH ejects of order its own mass before either coalescing in a burst of gravitational radiation, or, if the supply of ejectable matter runs out, stalling at a separation of $\sim 0.01-1 \mathrm{pc}$ [25].

The binary black hole model does a credible job of explaining the central density profiles of bright galaxies. Figure 2 plots the mass deficit[26]:

$$
M_{\text {def }} \equiv 4 \pi \int_{0}^{r_{b}}\left[\rho_{*}\left(r_{b}\right)\left(\frac{r}{r_{b}}\right)^{-\gamma_{0}}-\rho_{*}(r)\right] r^{2} d r
$$

the difference in the integrated mass between the observed density profile, and a $\rho_{*}(r) \propto r^{-\gamma_{0}}$ profile extrapolated inward from the turnover radius $r_{b}$. There is a good correlation of $M_{\text {def }}$ with $M_{\bullet}$, although the constant of proportionality can lie anywhere between $\sim 1$ and $\sim 10$ depending on the choice of $\gamma_{0}$. Larger ratios would be consistent with a picture in which galaxies form through a succession of mergers 25].

Interestingly, another class of stellar system exhibits both spikes and cores: the globular clusters. Globular clusters may contain "intermediate mass" $\left(M_{\bullet} \sim 10^{3} M_{\odot}\right)$ black holes, although the evidence 27] is not compelling. However they almost certainly contained an early population of $\sim 10 M_{\odot}$ black holes, remnants of the first generation of stars [28, which would have spiralled to the center and displaced the lighter stars before ejecting themselves via the gravitational slingshot. This mechanism appears capable of creating the cores [29]. The steep central spikes seen in some globular clusters [30] are probably a consequence of collisional relaxation. 


\section{Implications for Dark Matter Spikes}

A dark matter spike could have survived at the center of the Milky Way if no mergers occurred since the era of SBH formation[31. But the formation of the SBH was itself probably triggered by a major merger which channeled gas into the central regions [19]; indeed a binary SBH may have played a crucial role in the channeling [32]. It is hard to see how a dark matter spike could have survived such an event, and in fact the quasistationary conditions critical for the formation of the spike may never have existed [33]. These arguments suggest that a dark matter spike is unlikely at the center of the Galaxy, even if the Milky Way disk has not suffered a major merger in the last $\sim 12$ Gyr [34]. A conservative upper limit on the density of neutralinos at the Galactic center would be that given by CDM models of structure formation, ignoring the SBHs [22]. Spikefree density profiles might still allow testing of significant portions of MSSM parameter space 35 .

\section{Acknowledgments}

This work was supported by NSF grants AST 00-71099 and AST 02-06031, by NASA grants NAG5-6037 and NAG5-9046, and by grant HST-AR-08759 from STScI. 
[1] J. L. Feng, K. T. Matchev and F. Wilczek, Phys. Rev. D 63, 045024 (2001).

[2] V. Berezinsky, A. Bottino and G. Mignola, Phys. Lett. B 325, 136 (1994).

[3] L. Bergstrom, P. Ullio and J. H. Buckley, Astropart. Phys. 9, 137 (1998).

[4] P. Gondolo and J. Silk, Phys. Rev. Lett. 83, 1719 (1999).

[5] P. J. E. Peebles, Gen. Rel. Grav. 3, 61 (1972).

[6] P. Young, Astron. J. 242, 1232 (1980).

[7] D. Merritt and G. Quinlan, Astrophys. J. 498, 625 (1998).

[8] J. F. Navarro, C. S. Frenk and S. D. M. White, Astrophys. J. 462, 563 (1996).

[9] B. Moore et al., Astrophys. J. 499, L5 (1999).

[10] G. D. Quinlan, L. Hernquist and S. Sigurdsson, Astrophys. J. 440, 554 (1995).

[11] C. Power et al., Mon. Not. R. Astron. Soc. 338, 14 (2003).

[12] B. Moore, astro-ph/0103100 (2001).

[13] S. Faber et al., Astron. J. 114, 1771 (1997).

[14] D. Merritt and T. Fridman, in "Fresh Views of Elliptical Galaxies," ASP Conf. Ser. Vol. 86, ed.

A. Buzzoni, A. Renzini \& A. Serrano (Astron. Soc. Pac.), 13 (1996).

[15] K. Gebhardt et al., Astron. J. 112, 105 (1996).

[16] D. Merritt, in "Coevolution of Black Holes and Galaxies," ed. L. C. Ho (astro-ph/0301257) (2003).

[17] R. Bender, in "The Nature of Elliptical Galaxies," ASP Conf. Ser. Vol. 116, ed. M. Arnaboldi, G.

S. Da Costa \& P. Saha (Astron. Soc. Pac.), 11 (1997).

[18] E. Serabyn and M. Morris, Nature 382, 602 (1995).

[19] G. Kauffmann and M. Haehnelt, Mon. Not. R. Astron. Soc. 311, 576

[20] T. Lauer et al., Astron. J. 124, 1975 (2002).

[21] D. Merritt and F. Cruz, Astrophys. J. 551, 41 (2001).

[22] D. Merritt, M. Milosavljevic, L. Verde and R. Jimenez, Phys. Rev. Lett. 88, 191301 (2002).

[23] W. C. Saslaw, M. J. Valtonen and S. J. Aarseth, Astrophys. J. 190, 253 (1974).

[24] G. D. Quinlan, New Astron. 1, 35 (1996).

[25] M. Milosavljevic and D. Merritt, Astrophys. J., 563, 34 (2001).

[26] M. Milosavljevic, D. Merritt, A. Rest and F. C. van den Bosch, Mon. Not. R. Astron. Soc. 331, 51 (2002).

[27] K. Gebhardt, R. M. Rich and L. C. Ho, Astrophys. J. 578, 41 (2002).

[28] S. F. Portegies Zwart and S. L. W. McMillan, Astrophys. J. 528, L17 (2000).

[29] D. Merritt and M. Milosavljevic, in "Dark Matter in Astro- and Particle Physics," ed. H. V. Klapdor-Kleingrothaus \& R. D. Viollier, 79 (2002).

[30] S. Djorgovski and I. R. King, Astrophys. J. 305, 61 (1986).

[31] G. Bertone, G. Sigl and J. Silk, Mon. Not. R. Astron. Soc. 337, 98 (2002).

[32] A. Gould and H. W. Rix, Astrophys. J. 532, 29 (2000).

[33] P. Ullio, H. Zhao and M. Kamionkowski, Phys. Rev. D 64, 3504 (2001).

[34] R. F. G. Wyse, in ASP Conf. Ser. Vol. 230, "Galaxy Disks and Disk Galaxies," ed. J. G. Funes, S. J. \& E. M. Corsini, (Astron. Soc. Pac.), 71 (2001).

[35] P. Ullio, J. High Energy Phys. 2001, 53 (2001). 